\title{
Carbapenem-Resistant Enterobacteriaceae: Analyzing Knowledge and Practice in Healthcare Providers
}

Background: Gram negative antibiotic resistance is increasing worldwide as both Carbapenem-Resistant Enterobacteriaceae (CREs) and Enterobacteriaceae producing extended spectrum B-lactamases (ESBLs) become more common. Objective: We analyzed clinicans' knowledge regarding resistant gram-negative organisms with respect to infection control practices, prescribing practices and assessment of their patients' risk for resistant infections. Design: Online survey Participants: Target population included clinicians who prescribe antibiotics i.e. medical doctors and mid-level practitioners, at three Massachusetts hospitals. Methods: Questionnaires were sent to 3 Tufts-affiliated teaching hospitals to assess level of knowledge and elucidate perceptions about gram- negative resistance. Results: We received 434 responses from 3332 non-infectious disease clinicians (13\%) surveyed at the three hospitals. $51.1 \%$ of clinicians correctly scored $50 \%$ or greater on the knowledge questions. Internal medicine clinicians had higher knowledge scores than noninternal medicine clinicians ( $62 \%$ vs $45 \%$; OR=1.67, $p=0.02$ ). Clinicians within three years of training had higher scores than those with more than 10 years of training $(64.3 \%$ vs $44 \%$ $\mathrm{OR}=2.3, \mathrm{p}=0.002)$. Clinicians with fewer years since training and those with higher knowledge scores were more likely to appropriately consider certain patients at risk for resistant infections $(p<0.05) .54 .4 \%$ of clinicians were very concerned about gram-negative antibiotic resistance. $64.6 \%$ of clinicians felt comfortable de-escalating antibiotics as cultures are available. Conclusion: We found overall low knowledge scores and much variability in the way clinicians assess whether certain patient populations are at risk for antibiotic resistance. Internal medicine clinicians and those with fewer years since completion of their training scored higher and more appropriately considered patients at risk for resistance. The majority of clinicians are concerned about gram-negative resistance and indicated they would de-escalate antibiotic therapy if they had susceptibility information. These results will help focus and target our teaching and awareness-raising strategies. [b] 0 
2 Carbapenem-Resistant Enterobacteriaceae: Analyzing Knowledge and Practice in Healthcare 3 Providers

4 Evangeline Thibodeau, MD, $\mathrm{MPH}^{1}$, Shira Doron, $\mathrm{MD}, \mathrm{MS}^{1}$, Vito Iacoviello, $\mathrm{MD}^{2}$

5 Jennifer Schimmel $\mathrm{MD}^{3}$, David R. Snydman, $\mathrm{MD}^{1}$

6 (1) Division of Geographic Medicine and Infectious Diseases, Tufts Medical Center and Tufts

7 University School of Medicine, Boston, MA, USA (2) Saint Elizabeth's Medical Center,

8 Brighton, MA, USA (3) Baystate Medical Center, Springfield, MA, USA

9 Corresponding Author:

10 Shira Doron

11800 Washington St.

$12 \quad \# 238$

13 Boston, MA 02111

14 sdoron@tuftsmedicalcenter.org

15 617-636-2868

16 Fax: 617-636-8525

17 Running Title: CRE Survey to Hospital Clinicians

18 Text word count: 1871

19 Abstract word count: 297

20 Number of tables: 1

21 Number of figures: 2

22 Number of references: 17

23 Key words: Antibiotic resistance, Provider knowledge, multidrug-resistant organisms 


\section{Abstract}

25 Background: Gram negative antibiotic resistance is increasing worldwide as both Carbapenem26 Resistant Enterobacteriaceae (CREs) and Enterobacteriaceae producing extended spectrum B-

27 lactamases (ESBLs) become more common.

28 Objective: We analyzed clinicans' knowledge regarding resistant gram-negative organisms with respect to infection control practices, prescribing practices and assessment of their patients' risk

30 for resistant infections.

31 Design: Online survey

32 Participants: Target population included clinicians who prescribe antibiotics i.e. medical doctors 33 and mid-level practitioners, at three Massachusetts hospitals.

34 Methods: Questionnaires were sent to 3 Tufts-affiliated teaching hospitals to assess level of

35 knowledge and elucidate perceptions about gram- negative resistance.

36 Results: We received 434 responses from 3332 non-infectious disease clinicians (13\%) surveyed

37 at the three hospitals. $51.1 \%$ of clinicians correctly scored $50 \%$ or greater on the knowledge

38 questions. Internal medicine clinicians had higher knowledge scores than non-internal medicine

39 clinicians $(62 \%$ vs $45 \%$; OR=1.67, $\mathrm{p}=0.02)$. Clinicians within three years of training had higher

40 scores than those with more than 10 years of training $(64.3 \%$ vs $44 \%$ OR $=2.3, p=0.002)$.

41 Clinicians with fewer years since training and those with higher knowledge scores were more

42 likely to appropriately consider certain patients at risk for resistant infections $(\mathrm{p}<0.05) .54 .4 \%$ of

43 clinicians were very concerned about gram-negative antibiotic resistance. $64.6 \%$ of clinicians felt

44 comfortable de-escalating antibiotics as cultures are available.

45 Conclusion: We found overall low knowledge scores and much variability in the way clinicians

46 assess whether certain patient populations are at risk for antibiotic resistance. Internal medicine

47 clinicians and those with fewer years since completion of their training scored higher and more

48 appropriately considered patients at risk for resistance. The majority of clinicians are concerned 
49 about gram-negative resistance and indicated they would de-escalate antibiotic therapy if they

50 had susceptibility information. These results will help focus and target our teaching and

51 awareness-raising strategies. 


\section{Background}

54 positive organisms such as Methicillin-resistant Staphylococcus aureus (MRSA), concern is

55 growing regarding more extensive antimicrobial resistance in gram-negative organisms.

56 Carbapenems, including imipenem, ertapenem, meropenem, and now doripenem, have been used

57 increasingly over the past decade to treat infections due to Enterobacteriaceae producing

58 extended spectrum ß-lactamases (ESBLs). Emergence of carbapenem-resistant

59 Enterobacteriaceae (CRE) is worrisome, particularly since there are limited antibiotic options to treat such infections, many of which are associated with significant adverse events. (1) Not only are drug options limited, it has been shown that patients infected with CREs suffer a 3-fold increased mortality compared to patients with infection due to a susceptible strain. (2)

While infection control practices have been shown to decrease the spread of resistance during outbreaks (3), given the limited antibiotic choices to treat these infections, awareness and prevention by clinicians is imperative in preventing further spread of this epidemic. Five-hundred and three physicians were surveyed in a University in France regarding antimicrobial resistance: $98 \%$ of physicians identified antimicrobial resistance as a national problem, yet only $74 \%$ of surveyed physicians felt it affected their daily practice. Interns reported more training in antibiotic resistance than senior physicians (59\% vs 34\%). (4) This study focused on MRSA, a gram positive organism, rather than gram-negative resistance such as ESBLs or CREs. There is a need to better understand the general knowledge and practice of healthcare practitioners in relation to the more recent and rapidly evolving gram-negative resistance problem. This information can be used so antimicrobial stewardship teams and infection disease specialists may identify knowledge gaps and inappropriate practices to better focus their educational efforts. With improved education regarding appropriate risk assessment and prescribing practice, the further development of resistance may be slowed. 


\section{$\underline{\text { Methods }}$}

8

We conducted an online survey using the SurveyMonkey $\square$ platform to better understand the knowledge and practice of health care practitioners regarding resistant gram-negative organisms. Responses were collected from September, 2011 through January 2012. The survey was sent to three hospitals in the Boston, Massachusetts metropolitan area: Tufts Medical Center, Saint Elizabeth's Medical Center, and Baystate Medical Center. Each hospital is an academic institution affiliated with Tufts Medical School. Tufts Medical Center is a 415 bed tertiary care center located in downtown Boston. Saint Elizabeth's Medical Center is 272 bed hospital located in a Boston suburb. Baystate Medical Center is a 716 bed facility located in western Massachusetts. During the year of the survey, in 2011, the rate of ESBL identification amongst isolates of Escherichia coli, Klebsiella oxytoca, and Klebsiella pneumoniae at these three hospitals ranged from 3-6\% and there were no CREs identified. The survey was approved by all hospitals' respective International Review Boards. Consent was inferred when a subject completed the survey. The survey was sent electronically by email list serves, accessed through the respective employee affairs' offices. In order to increase survey response, participants were entered into a raffle to win an Amazon gift card. Data was analyzed using SPSS for frequency tables and SAS 9.2 for multivariable logistic regression. Please see appendix for the survey in its entirety.

\section{$\underline{\text { Results }}$}

The survey was sent to 3332 clinicians at the three hospitals and 434 (13\%) complete responses were received. Note 15 responses from Infectious Disease physicians were removed leaving 419 responses for analysis (194 from Tufts Medical Center, 177 from Baystate Medical

Center and 48 from Saint Elizabeth's Medical Center). The survey was analyzed in the 5 following sections (in order of taking the survey): knowledge, opinion, risk perception, 101 prescribing practices, review practices. 
The characteristics of the respondents are displayed in Table 1.

\section{Knowledge assessment}

104

Knowledge questions were analyzed using a composite score from the six survey

105 knowledge questions: respondents were divided into those that scored $50 \%$ or higher on the

106 composite score and those that did not. Overall, $51.1 \%$ of clinicians scored $50 \%$ or higher on the

107 knowledge questions (range $0 \%$ to $100 \%$ ). However, $62 \%$ of internal medicine (IM) trained

108 clinicians scored $50 \%$ or higher on their composite knowledge score compared to $45 \%$ of non-IM

109 trained clinicians. $(\mathrm{OR}=1.67 \mathrm{p}=0.02)$ In addition, a significantly larger percentage of clinicians

110 who were within 3 years since completion of their training scored $50 \%$ or higher on their

111 composite knowledge score compared to practitioners with $>10$ years of experience (figure 1,

$112 \mathrm{OR}=2.3, \mathrm{p}=0.002$ ); there was no difference in higher scores between physicians within 3 years

113 since completion compared with clinicians within 3-10 years since completion $(\mathrm{p}=0.85)$.

\section{$114 \quad$ Opinion assessment}

The majority of respondents were concerned about gram-negative resistance with $54.4 \%$

very concerned, and just 3.6\% not knowing about gram-negative resistance prior to taking the

118 are sufficient new antibiotics in development to treat resistant gram negative infections", however

$11921.6 \%$ of clinicians reported having "no idea" what their opinion was regarding the statement.

\section{Risk perception assessment}

The majority of clinicians correctly identified patients at risk for resistant infections:

122 dialysis patients (69.5\%), patients residing in nursing homes (66.9\%), and patients admitted

123 within the past 30 days $(66.2 \%)$, however overall scores were still low (figure 2$)$. There was a

124 correlation between knowledge scores and risk consideration with a higher proportion of

125 clinicians who scored higher on the knowledge questions correctly considering patients at risk for

126 resistance compared with clinicians who scored lower on the knowledge questions. For example, 
$12766.8 \%$ of clinicians with higher knowledge scores correctly identified dialysis patients at risk for

128 resistance compared with $51 \%$ of clinicians with lower knowledge scores (OR $1.6 \mathrm{p}=0.02)$. This

129 pattern was also demonstrated in clinicians recently completing training; clinicians within 3 years

130 of completing training were more likely to correctly identify patients at risk for infections

131 compared to clinicians more than 10 years out of training. There was no difference in risk

132 consideration practice between clinicians within 3 years since training completion compared with

133 those within 3-10 years since training completion, except when considering nursing home

134 patients

135 Prescribing practices

136 When prescribing antibiotics, $81.5 \%$ of clinicians consider if their patients are risk for

137 resistant infections at least half the time; only $1.9 \%$ of clinicians never consider if their patients

138 are at risk for resistant infections. The most common means of risk consideration is to review

139 prior microbiology reports ( $77.5 \%$ of clinicians) followed by consideration of recent contact with

140 healthcare environment (67\%), review of prior antibiotic exposures (65\%), and review of prior

141 notes or discharge summaries (50.2\%). In addition, $71.2 \%$ of clinicians call a specialist such as

142 infectious disease or antimicrobial management teams less than half the time when prescribing

143 antibiotics, and only $2 \%$ always call a specialist when prescribing antibiotics.

$144 \quad$ Review practices

145 When prescribing antibiotics, the majority of clinicians review antimicrobial history.

146 While $86.4 \%$ of clinicians review antimicrobial reports pertaining to the current infection at least

$14750 \%$ of the time, $62.4 \%$ of practitioners review antimicrobials reports that do not pertain to the

148 current infection (old reports) at least $50 \%$ of the time. The most common reason for not

149 reviewing the reports was when the records are not available.

150 When reviewing microbiological records, the majority of clinicians (57.3\%) reported

151 using the susceptibility designation ("susceptible/intermediate/resistant") to determine what 
152 antimicrobial to order, and only $34.4 \%$ of clinicians reported looking at mean inhibitory

153 concentration (MIC) values.

154 In addition, $64.6 \%$ of clinicians felt comfortable de-escalating antibiotics as cultures are 155 available.

\section{Discussion}

157 In the United States at least two million people are infected with resistant organisms 158 resulting in 23,000 deaths, with most deaths occurring in healthcare settings. (5) Given health 159 care practitioners are at the cornerstone of antibiotic prescribing, caring for patients admitted to 160 the hospital, and assessing their risk for resistant infections, it is imperative to understand 161 clinicians' baseline knowledge, prescribing practices, and risk assessment strategies. In this

162 analysis we found that overall, health care practitioners in internal medicine were more 163 knowledgeable about ESBL and CRE definitions and treatments compared to clinicians not 164 employed in internal medicine. We also found that clinicians with fewer years since completion 165 of their training had more knowledge than clinicians farther out from training.

166 It appears adequate concern exists regarding gram-negative resistance, however, there is

167 still a large proportion of clinicians that are not aware about the lack of new drugs in 168 development to treat such infections.

169 While the majority of clinicians consider if their patients are at risk for resistant infections 170 prior to prescribing antibiotics, and use the appropriate means to consider patients' risk, the type 171 of patients they consider at risk is variable. Most clinicians appropriately considered dialysis 172 patients, nursing home patients, and patients admitted within the previous 30 days at risk for

173 resistant infections, however, higher knowledge scores and fewer years of experience correlated

174 with appropriate consideration of some groups of patients at risk for resistant infections. This 175 validates our findings that not only are internal medicine trained clinicians and those with fewer 
176 years since training completion more knowledgeable about ESBL and CRE definitions, they are

177 more appropriately considering the patients at risk for these resistant infections.

178 When reviewing reports, clinicians often review reports pertaining to the current

179 infection, but do not review old reports sufficiently. In addition, the majority of clinicians do not

180 interpret MIC values, which would not be expected from a non-infectious disease specialist.

181 However, given the majority of clinicians do not call a specialist when prescribing antibiotics and

182 feel comfortable de-escalating antibiotics, perhaps educational efforts should be directed toward 183 MIC interpretation.

184 Limitations include the survey sampled. While previous studies have demonstrated $>50 \%$

185 of hospital is Massachusetts have identified CREs (6), higher rates exist in areas such as New

186 York City. While we would expect higher levels of awareness in such areas, given the increasing

187 concern for resistance and rapid spread, it is important to educate early and prior to resistance 188 rates reaching epidemic thresholds.

189 In summary, we found many gaps in knowledge, and a great deal of variability in 190 opinions, and risk assessment practices, in health care practitioners in regards to resistant gram-

191 negative infections. While it is not surprising that internal medicine employees performed better

192 overall, with higher knowledge scores and more appropriate risk perception, it is enlightening

193 that clinicians with fewer years since training completion fared better in many areas than

194 clinicians with more years of experience, which likely relates to the relatively new and increasing 195 concern for antibiotic resistance. The information from this survey will help focus and target our 196 teaching and awareness-raising strategies through existing programs such as orientation teachings 197 sessions, resident and attending targeted education conferences, and weekly emails. 
199 This study was supported by NIH Training Grant 5T32AI007329-17 and was supported in part by a 200 research grant from the Investigator-Initiated Studies Program of Merck Sharp \& Dohme Corp. The

201 opinions expressed in this paper are those of the authors and do not necessarily represent those of Merck 202 Sharp \& Dohme Corp.

\section{Acknowledgements}

204 Prior presentation: Presented at the $50^{\text {th }}$ annual Meeting of the Infectious Diseases Society of 205 America, San Diego, October 23, 2012.

\section{Conflicts of Interest}

207 Shira Doron is on the speakers' bureau for Optimer, Forest and Merck, has received research 208 funding from Merck, and has received consulting fees from Durata.

209 Vito Iacoviello was an HIV clinical consultant for Gilead Pharmaceuticals.

210 David Snydman is on the speaker's bureau for Merck, Cubist and Genentech, has received

211 research funds from Merck, Cubist, Forest, Astra Zeneca, Replidyne, Optimer, Pfizer and

212 Genentech, has been a consultant for CSL Behring, Genentech, Millenium, Genzyme, Boeringer

213 Ingelheim, Massachusetts Biologic Public Health Laboratories, Merck and Microbiotix, and has

214 provided expert testimony on behalf of Roche.

\section{References}

216 1. Nordmann P, Cuzon G, Naas T. The real threat of Klebsiella pneumoniae carbapenemase217 producing bacteria. Lancet Infect Dis 2009; 9:228-36.

218 2. Patel G, Huprikar S, Factor SH, Jenkins SG, Calfee DP. Outcomes of carbapenem-resistant

219 Klebsiella pneumoniae infection and the impact of antimicrobial and adjunctive therapies. Infect

220 Control Hosp Epidemiol 2008; 29:1099-106. 
221 3. Munoz-Price LS, Hayden MK, Lolans K, Won S, Calvert K, Lin M, Stemer A, Weinstein RA.

222 Successful control of an outbreak of klebsiella pneumoniae carbapenemase-producing K.

223 pneumoniae at a long-term acute care hospital. Infect Control Hosp Epidemiol. 2010;31(4):341-

224347.

225 4. Naqvi A, Pulcini C. Bacterial resistance and antibiotic prescription: a survey of hospital

226 physician perception, attitude, and knowledge. Med Mal Infect 2010 Nov;40(11):625-631.

227 5. Center for Disease Control and Prevention. Antibiotic/Antimicrobial Resistance; Threat Report

228 2013. Available at: http://www.cdc.gov/drugresistance/threat-report-2013/ Accessed October 1, 2292013

230 6. Thibodeau E, Duncan R, Snydman DR, Bolstorff B, Vostok J, Barton K, Demaria A Jr., 231 Carbapenem-Resistant Enterobacteriaceae (CRE): A Statewide Survey of Detection in

232 Massachusetts Hospitals. Infect Control Hosp Epidemiol. 2012 sEP;33(9):954-6. 


\section{Table 1 (on next page)}

Characteristics of survey respondents 


\section{Table 1: Characteristics of Survey Respondents ( $N=419)$}

Characteristic No. (\%)

Hospital

Tufts Medical Center

$194(46.3)$

Baystate Medical Center 177 (42.2)

Saint Elizabeth's Medical Center 48 (11.5)

MD/DO

$372(88.8)$

\section{Years of Practice}

$<3$

$129(30.8)$

3 to 10

$106(25.3)$

$>10$

$184(43.9)$

\section{Specialty*}

Internal Medicine

$213(51.1)$

Surgery

$46(11)$

Pediatrics

$67(16.1)$

$\mathrm{Ob} / \mathrm{Gyn}$

$26(6.2)$

Other (Psychiatry, Neurology,

Radiology, Anesthesia,

Radition/Oncology, Emergency

Medicine, PMR)

$65(15.6)$

*Note two missing values from specialty $(\mathrm{N}=417)$ 


\section{Figure 1}

Proportion of healthcare practitioners with knowledge scores $>50 \%$, by years of experience

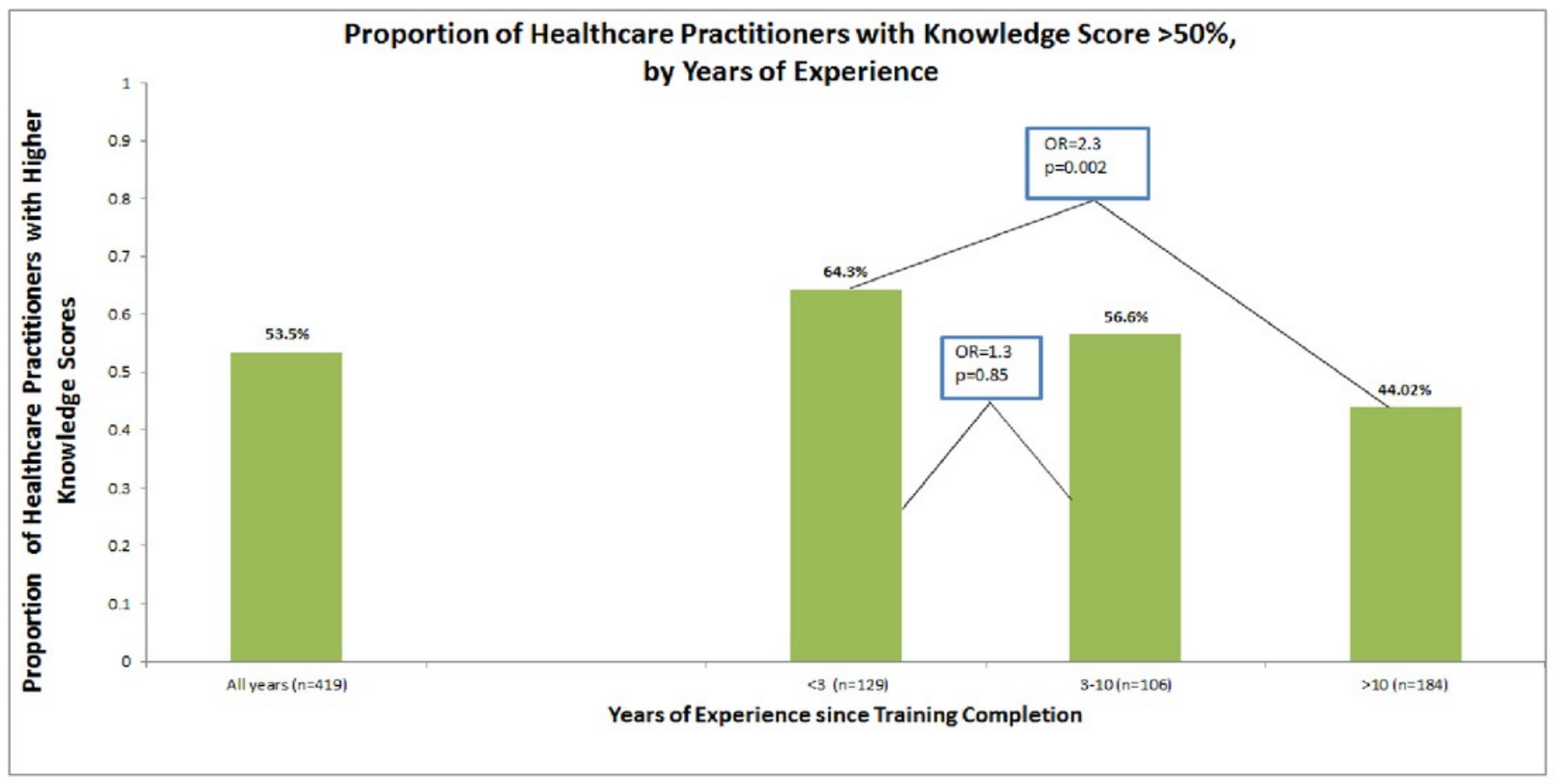


Antibiotic resistance is increasing worldwide. Much focus has been on gram-positive organism resistance. We are interested in the recently recognized rise in gram-negative organism resistance and therefore are conducting a survey of all physicians, nurse practitioners, and physician assistants regarding their knowledge of and practice regarding such organisms at Tufts Medical Center and four other Tufts-associated hospitals. While participation in this survey is voluntary, your responses will be used to research, prevent, and treat resistant organisms. The survey consists of 20-25 questions, depending on your responses, and will take you between 10-12 minutes to complete. In addition, by providing your email in a separate link, so to keep your responses anonymous, your name will be entered into a raffle to win one of three $\$ 150$ Amazon gift card

Please email any questions or comments to Evangeline Thibodeau at Ethibodeau@tuftsmedicalcenter.org

Thank you for your time and participation in this project.

1) What is your professional title?
a) MD
b) $\mathrm{DO}$
c) NP
d) PA
e) other: please specify

2) What year did you finish your education? For example, when did you graduate medical school or finish your NP/PA training?

3) Since completing your education, how many years have you been practicing your current specialty?

4) What department do you currently work in?
a) Internal Medicine
b) Internal Medicine subspecialty: please specify
c) Surgery
d) Surgical subspecialty: please specify
e) Pediatrics
f) Pediatrics subspecialty: please specify
g) Psychiatry
h) Obstetrics/Gynecology
i) Neurology
j) Other: please specify

5) Since finishing your education, have you worked in other specialties other than your current department?

a) No, I have only practiced in my current department

b) Yes 
6) If yes to question 5, what other specialties have you previously worked in? (check all that apply)
a) Internal Medicine
b) Internal Medicine subspecialty: please specify
c) Surgery
d) Surgical subspecialty: please specify
e) Pediatrics
f) Pediatrics subspecialty: please specify
g) Psychiatry
h) Obstetrics/Gynecology
i) Neurology
j) Other: please specify

The following questions are to assess your knowledge and practice regarding treatment of resistant gram-negative organisms. We expect that various areas of expertise and level of training will affect your knowledge of and experience with treating such infections. However to better understand the gaps in knowledge and where to focus our teaching efforts, we would like you to answer the following questions as honestly as possible. The results of this survey in no way will be graded or identified. They will be kept confidential and only a summary of the results will be reported in a scientific nature.

7) What is an extended spectrum beta-lactamase (ESBL)-producing organism?

a) An organism that is resistant to Vancomycin

b) An organism that carries an enzyme conferring resistance to most beta-lactam antibiotics including penicillins, cephalosporins, and monobactams such as aztreonam

c) An organism that produces an enzyme conferring resistance to all quinolone antibiotics.

d) An organism that is resistant to all cephalosporins except the $4^{\text {th }}$ generation cephalosporins such as cefipime but is sensitive other beta-lactam antibiotics such as monobactams (i.e. aztreonam)

e) An organism that produces an enzyme that makes it is sensitive only to Daptomycin

f) Do not know

8) What are Carbapenem Resistant Enterobacteriaceae (CRE)?

a) Organisms that produces an enzyme conferring resistance to both Vancomycin and Daptomycin

b) Organisms that produce an enzyme conferring resistance to all cephalosporins and all quinolones

c) Organisms that produces an enzyme conferring resistance to most B-lactam antibiotics including penicillins, cephalosporins, and monobactams such as aztreonam.

d) Organisms that produces an enzyme conferring resistance to all ß-lactam 
molecules including carbapenems such as Meropenem and Ertapenem.

e) Do not know

9) What is an example of a CRE?
a) New Delhi Metallo-protease (NDM)
b) Athens betalactamase (ABL)
c) Klebsiella producing carbapenamase (KPC)
d) $b$ and $c$
e) a and c
f) all of the above
g) Do not know

10) If your patient was infected with an ESBL, what antibiotic or class of antibiotics would be most appropriate therapy? (check all that apply)
a) $4^{\text {th }}$ Generation cephalosporins such as cefipime
b) Quinolones
c) Tigecycline
d) Daptomycin
e) Carbapenems such as Meropenem or Ertapenem
f) Colistin
g) Do not know

11) If your patient was infected with a CRE, what antibiotic or class of antibiotics would be most appropriate therapy? (check all that apply)
a) $4^{\text {th }}$ Generation cephalosporins such as cefipime
b) Quinolones
c) Tigecycline
c) Daptomycin
e) Carbapenems such as Meropenem or Ertapenem
f) Colistin
g) Do not know

12) Which of the following statements best describes your opinion regarding gram negative resistance?
a) I didn't know there was gram-negative resistance until I took this survey
b) I am not concerned about gram negative resistance
c) I am a little concerned about gram-negative resistance
d) I am moderately concerned about gram-negative resistance
e) I am very concerned about gram-negative resistance

13) How strongly do you agree with the following statement: "There are sufficient new antibiotics in development to treat resistant gram negative infections:
a) I do not agree with this statement at all
b) I agree with this statement a little
c) I agree with this statement a lot
d) I agree with this statement completely 
e) I have no idea

14) Prior to prescribing or ordering any antibiotic how often do you consider whether your patient is at risk for a resistant organism?
a) never
b) sometimes but less than half of the time
c) about half of the time
d) more often than not
e) always
f) I have never prescribed or ordered an antibiotic

15) If yes to 14 (answer b-e), how do you determine if they are at risk? (check all that apply)

a) Review prior microbiological reports

b) Review prior antibiotic exposures

c) Review prior discharge summaries and outpatient notes

d) Consider recent contact with the healthcare environment

e) Other: please explain

16) What patients or populations do you consider at risk for resistant organisms? (check all that apply)
a) Patients on hemodialysis
b) Patients who reside in a nursing home
c) Patients who have received antibiotics in the past year
d) Patients who have been admitted to the hospital within 30 days
e) Community-residing patients who have not received antibiotics within 90 days
f) Immunocompromised patients
g) Patients in the ICU
h) None of the above
i) All of the above

17) Prior to prescribing an antibiotic for your patient how often do you review their antimicrobial history that does not pertain to the current infection?
a) never
b) sometimes but less than half of the time
c) about half of the time
d) more often than not
e) always

18) When you do not always review the antimicrobial history (answer a-d to 17), what are the reasons? (check all that apply)
a) I don't know how to interpret them
b) Not enough time
c) I always call a specialist to help me when I need it
d) When the records are not available
e) I do not consider them relevant 
f) Other: please explain

19) Prior to prescribing an antibiotic for your patient how often do you review the antimicrobial history for the current infection?
a) never
b) sometimes but less than half of the time
c) about half of the time
d) more often than not
e) always

20) When you do not always review the current record (answer a-d to 19), what are the reasons? (check all that apply)
a) I don't know how to interpret them
b) Not enough time
c) I always call a specialist to help me
d) When the records are not available
e) I do not consider them relevant
e) Other: please explain

21) When you review the microbiological records, which of the following methods do you use most often?

a) I never review the microbiological reports

b) I look at the drugs that are reported as sensitive and use those

c) I look at the MIC cutoffs to determine which drugs are sensitive

d) I look at the MIC cutoffs of each drug and see if it is appropriate for the bacteria I want to treat

e) I call the micro lab to discuss the data

f) Other: please explain

22) If you have started empiric antibiotics, prior to knowing results of the culture data, what best describes your practice of deescalating or targeting antibiotics when the culture and susceptibility report is later available?

a) I never deescalate or target antibiotics

b) I call for help from a specialist such as an ID consult or the Antimicrobial Management Team (AMT) the majority of the time

c) I feel fairly comfortable reviewing the report and changing antibiotics myself the majority of the time

d) Other: Please explain

23) When prescribing an antibiotic for any reason, how often do you call a specialist such as an ID consult or AMT?
a) never
b) sometimes but less than half of the time
c) about half of the time
d) more often than not
e) always 
24) What is the main reason you call an ID or AMT consult when prescribing antibiotics (excluding the requirement for antimicrobial approval)?

Separate link for the following question:

25) Please provide your email address if you wish to enter into the raffle: 


\section{Figure 2}

Proportion of healthcare practitioners recognizing risk factors for resistance

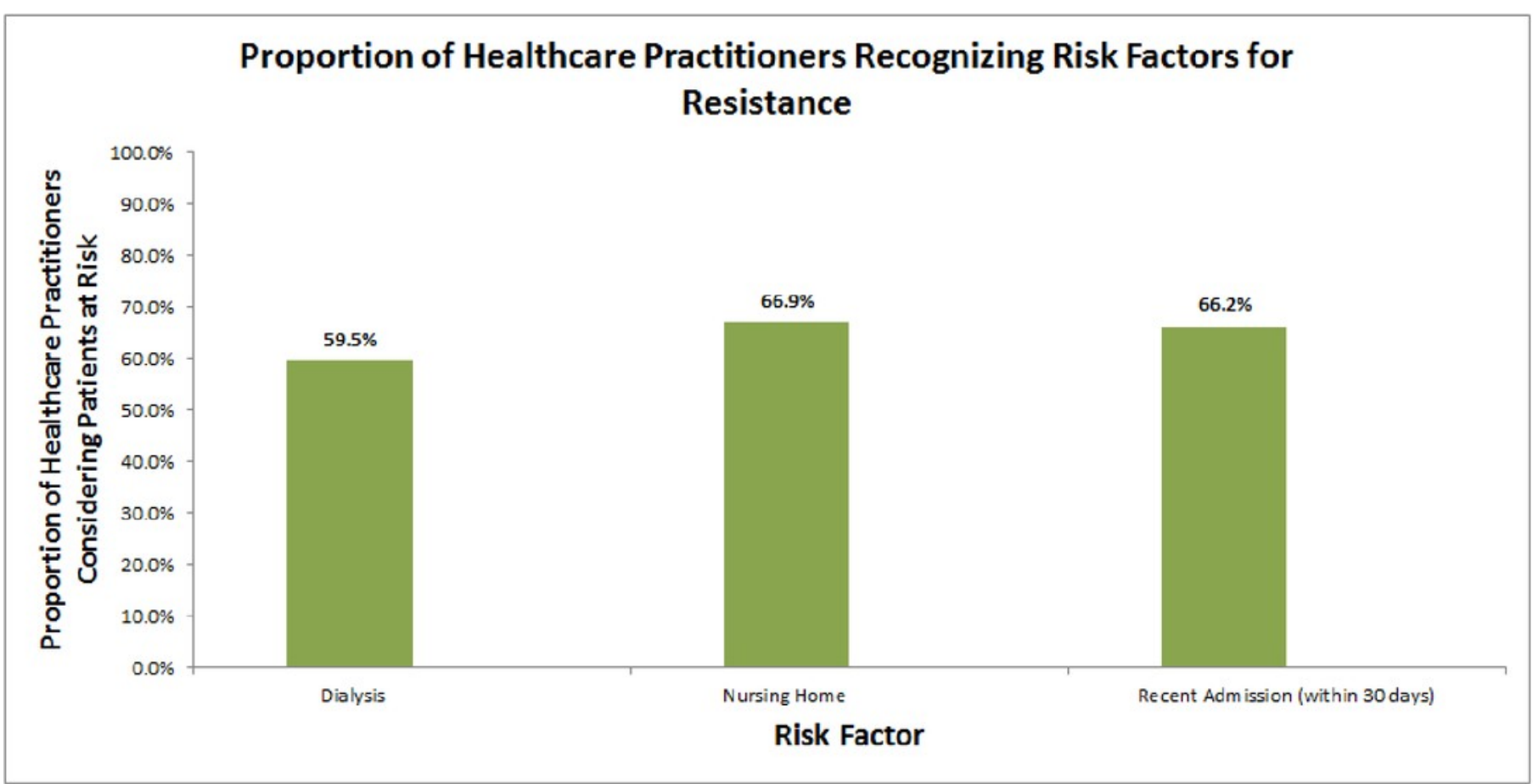

